\title{
EDITORIAL
}

\section{Neutrophils in chronic inflammatory airway diseases: can we target them and how?}

\author{
Y. Gernez*, R. Tirouvanziam ${ }^{\#}$ and P. Chanez
}

M any lung diseases, such as severe asthma, chronic obstructive pulmonary disease (COPD), cystic fibrosis $(\mathrm{CF})$, bronchiectasis and infiltrative pulmonary diseases are associated with chronic neutrophilic inflammation. These diseases are common, can be severe and, together, represent a major economic burden and worldwide public health challenge. In addition to chronic handicap, these diseases also feature recurrent episodes of acute worsening of inflammatory symptoms, known as exacerbations, a leading cause for progressive lung impairment. Prevention and prompt management of exacerbations is strongly recommended since they often influence disease prognosis.

In COPD [1], the high number of neutrophils recruited to the lungs is known to positively correlate with the thickness of the sputum [2]; to account, in large part, for the poor response to corticosteroid treatment [3]; and to associate with poorer prognosis [4]. Recruited neutrophils have been shown to infiltrate the airway epithelium and submucosal glands [2] from the proximal to the distal part of the airway tree.

Activation of lung neutrophils leads to the release of granule proteins, including human neutrophil elastase (HNE) and myeloperoxidase (MPO) [5]. HNE and MPO contribute to the bronchial inflammation and to structural changes such as peribronchiolar fibrosis and emphysema [6,7]. None of the currently available therapies has any effect on the release and/ or pathological activity of these cytotoxic enzymes. Until therapies efficient at curbing down neutrophil function are developed, lowering neutrophil recruitment may represent the best way to counteract their central role in airway inflammation in COPD.

Neutrophil recruitment to the airways is controlled by various mediators, most notably CXCL8 (also known as interleukin (IL)-8), IL-1 $\beta$, tumour necrosis factor (TNF)- $\alpha$, and leukotriene $B_{4}$. Hence, there is extensive functional redundancy in signalling pathways leading to airway recruitment of neutrophils (fig. 1). These signalling pathways are further complicated by the fact that ligand-receptor relationships are rarely exclusive. For example, CXCL8 signals through both low- and

Depts of *Allergy and Immunology and "Paediatrics, Stanford University School of Medicine Stanford, CA, USA. 'Département des Maladies Respiratoires, AP-HM, Laboratoire d'Immunologie INSERM CNRS U 600, UMR6212, Marseille, France.

CORRESPONDENCE: P. Chanez, Département des Maladies Respiratoires, AP-HM, Laboratoire d'Immunologie INSERM CNRS U 600, UMR6212, Marseille, France. E-mail: pascal.chanez@ univmed.fr high-affinity receptors designated CXCR1 and CXCR2, respectively. CXCL2 itself can respond to other chemokines beyond CXCL8, including CXCL1, 2, 3, 5, 6 and 7. In animal models for various pulmonary conditions, including COPD, blockade of CXCR1 and CXCR2 by specific inhibitors significantly reduced neutrophilic airway inflammation [8]. Both CXCR1 and CXCR2 are expressed in high levels in endobronchial biopsies from patients with severe COPD that are prone to exacerbate [9]. They also have been found in bronchial epithelium from healthy controls [10].

Neutrophils play an essential role for the antimicrobial response of the lungs, and CXCR2 inhibition has been shown, in animal models, to impair neutrophil function, especially their ability to kill infectious agents [11, 12]. However, in humans, it appears that CXCR1, rather than CXCR2, is the functionally important receptor subtype involved with neutrophil degranulation [13], therefore phagocytosis may remain preserved following anti-CXCR2 use.

These data have prompted several companies to develop CXCR2 inhibitors as potential anti-inflammatory therapy in patients with COPD or other conditions with chronic neutrophilic airway inflammation [14]. In the present issue of the European Respiratory Journal, Holz et al. [15] report the effect of SCH527123, a selective CXCR2 inhibitor tested in healthy volunteers as a modulator of neutrophilic airway inflammation following an experimental ozone challenge. In this study, SCH527123 treatment was found to significantly decrease

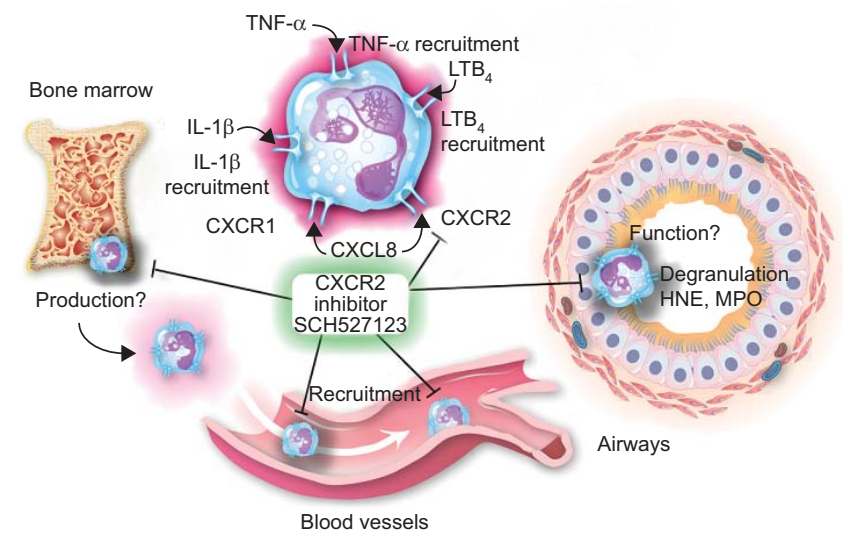

FIGURE 1. CXCR2 inhibitors in the context of airway neutrophilic inflammation. TNF: tumour necrosis factor; LT: leukotriene; IL: interleukin; HNE: human neutrophil elastase; MPO: myeloperoxidase. 
ozone-induced airway neutrophilia measured in the induced sputum (measured as \% and count per mL). Additionally, SCH527123 induced a significant decrease in CXCL8 and MPO levels (measured as protein levels, not activity). These results support the hypothesis that CXCR2 inhibition may have beneficial effects in COPD. Interestingly, treatment with AZD-8309, another CXCR2 inhibitor, resulted in a significant $(\sim 80 \%)$ reduction in total sputum cells and neutrophils compared with placebo after lipopolysaccharide challenge as well as a significant reduction in HNE activity in the sputum [16]. Besides, a monoclonal antibody against CXCL8, ABX-IL8, has been found to significantly reduce the severity of dyspnoea (measured with the transition dyspnoea index), compared with placebo, in patients with COPD $[17,18]$.

It has to be noted that the experimental inflammation by ozone challenge used in the study by HoLz et al. [15] to test the effect of SCH527123 is chiefly CXCL8-dependent, transient and fully reversible [19]. In contrast, the pathological inflammation occurring in the airways of patients with COPD depends on multiple mediators (fig. 1), is chronic and largely irreversible. Thus, even if CXCR2 inhibition can reduce neutrophil recruitment in the context of acute ozone-induced airway inflammation in healthy controls, the efficacy of CXCR2 in patients with complex chronic neutrophilic lung inflammation will need to be demonstrated in further studies.

HoLz et al. [15] also report that mild-to-moderate blood neutropaenia was observed in patients on active therapy with SCH527123. However, this adverse effect was transient and circulating neutrophil levels bounced back to baseline shortly after treatment cessation. In the future, this adverse effect should be monitored very carefully. This is particularly important in the context of COPD and severe asthma, in which an adequate neutrophil balance is key to combating recurrent bacteria-driven pneumonia and exacerbations [20].

So far, underlying mechanisms causing chronic airway neutrophilia in COPD remain unknown. It is possible that normal negative feedback loops regulating the scale and timely termination of beneficial neutrophilic responses upon airway infection are defective in COPD patients. Interestingly, neutrophils recruited to the airways of CF patients were recently found to escape their normal pro-apoptotic programme and secrete HNE and MPO actively [21]. Such dysfunction of live neutrophils, rather than their rapid demise by necrosis, may also play a role in COPD. Nonetheless, the study by HoLz et al. [15] raises hopes for better therapeutic management of neutrophilic airway inflammation. In the future, the use of CXCR2 inhibitors could certainly contribute to a better understanding of the role of neutrophils in COPD and other airway diseases.

Neutrophils are endowed with a wide collection of receptors to chemoattractants, including CXCR1 and CXCR2, respectively the low- and high-affinity receptors for CXCL8 (IL-8). SCH527123, the molecule tested in the study by HoLz et al. [15], is a specific CXCR2 inhibitor. Mature neutrophils exist in four distinct compartments [22]: 1) the bone marrow reservoir; 2) the circulating pool; 3) the marginated pool, composed of neutrophils tethered to the endothelial layer; and 4) the tissue pool, as shown here with airway neutrophils. CXCR2 is expressed on all four pools and, therefore, CXCR2 inhibitors are likely to impact all four pools, as illustrated.

\section{STATEMENT OF INTEREST}

A statement of interest for P. Chanez can be found at www.erj. ersjournals.com $/ \mathrm{misc} /$ statements.dtl

\section{REFERENCES}

1 Bourdin A, Burgel P-R, Chanez P, et al. Recent advances in COPD: pathophysiology, respiratory physiology and clinical aspects, including comorbidities. Eur Respir Rev 2009; 18: 198-212.

2 Saetta M, Turato G, Facchini FM, et al. Inflammatory cells in the bronchial glands of smokers with chronic bronchitis. Am J Respir Crit Care Med 1997; 156: 1633-1639.

3 Macdowell AL, Peters SP. Neutrophils in asthma. Curr Allergy Asthma Rep 2007; 7: 464-468.

4 Nadel JA. Role of neutrophil elastase in hypersecretion during COPD exacerbations, and proposed therapies. Chest 2000; 117: Suppl. 2, 386S-389S.

5 Borregaard N, Sorensen OE, Theilgaard-Monch K. Neutrophil granules: a library of innate immunity proteins. Trends Immunol 2007; 28: 340-345.

6 Ilumets H, Rytila PH, Sovijarvi AR, et al. Transient elevation of neutrophil proteinases in induced sputum during COPD exacerbation. Scand J Clin Lab Invest 2008; 68: 618-623.

7 Weiss SJ. Tissue destruction by neutrophils. N Engl J Med 1989; 320: 365-376.

8 Chapman RW, Minnicozzi M, Celly CS, et al. A novel, orally active CXCR1/2 receptor antagonist, SCH527123, inhibits neutrophil recruitment, mucus production, and goblet cell hyperplasia in animal models of pulmonary inflammation. J Pharmacol Exp Ther 2007; 322: 486-493.

9 Qiu Y, Zhu J, Bandi V, et al. Biopsy neutrophilia, neutrophil chemokine and receptor gene expression in severe exacerbations of chronic obstructive pulmonary disease. Am J Respir Crit Care Med 2003; 168: 968-975.

10 Gras D, Tiers L, Vachier I, et al. Regulation of CXCR/IL-8 in human airway epithelial cells. Int Arch Allergy Immunol 2009; 152: 140-150.

11 Banerjee K, Biswas PS, Kim B, et al. CXCR2 ${ }^{-/-}$mice show enhanced susceptibility to herpetic stromal keratitis: a role for IL-6-induced neovascularization. J Immunol 2004; 172: 1237-1245.

12 Del Rio L, Bennouna S, Salinas J, et al. CXCR2 deficiency confers impaired neutrophil recruitment and increased susceptibility during Toxoplasma gondii infection. J Immunol 2001; 167: 65036509.

13 Jones SA, Wolf $\mathrm{M}$, Qin $\mathrm{S}$, et al. Different functions for the interleukin 8 receptors (IL-8R) of human neutrophil leukocytes: NADPH oxidase and phospholipase D are activated through IL8R1 but not IL-8R2. Proc Natl Acad Sci USA 1996; 93: 66826686.

14 Balmes JR, Aris RM, Chen LL, et al. Effects of ozone on normal and potentially sensitive human subjects. Part I: Airway inflammation and responsiveness to ozone in normal and asthmatic subjects. Res Rep Health Eff Inst 1997; 78: 1-37.

15 Holz O, Khalilieh S, Ludwig-Sengpiel A, et al. SCH527123, a novel CXCR2 antagonist, inhibits ozone-induced neutrophilia in healthy subjects. Eur Respir J 2010; 35: 564-570.

16 O'Connor BJ, Leaker B, Barnes P, et al. Inhibition of LPS-induced neutrophilic inflammation in healthy volunteers. Eur Respir J 2007; 30: Suppl. 51, 209s.

17 Mahler DA, Weinberg DH, Wells CK, et al. The measurement of dyspnea. Contents, interobserver agreement, and physiologic correlates of two new clinical indexes. Chest 1984; 85: 751-758. 
18 Mahler DA, Huang S, Tabrizi M, et al. Efficacy and safety of a monoclonal antibody recognizing interleukin-8 in COPD: a pilot study. Chest 2004; 126: 926-934.

19 Aris RM, Christian D, Hearne PQ, et al. Ozone-induced airway inflammation in human subjects as determined by airway lavage and biopsy. Am Rev Respir Dis 1993; 148: 1363-1372.
20 Vestbo J. The TORCH (towards a revolution in COPD health) survival study protocol. Eur Respir J 2004; 24: 206-210.

21 Sabroe I, Whyte MK. Incapacitating the immune system in cystic fibrosis. Nat Med 2007; 13: 1417-1418.

22 Hogg JC, Walker BA. Polymorphonuclear leucocyte traffic in lung inflammation. Thorax 1995; 50: 819-820. 